\title{
Functionalization of Graphene Oxide Nanosheets Can Reduce Their Cytotoxicity to Dental Pulp Stem Cells
}

\author{
Ahmad Gholami $\mathbb{D}^{1},{ }^{1}$ Fatemeh Emadi $\left(\mathbb{D},{ }^{2}\right.$ Abbas Amini $\left(\mathbb{D},{ }^{3}\right.$ Mansoureh Shokripour $\mathbb{D}^{4},{ }^{4}$ \\ Mostafa Chashmpoosh $\left(\mathbb{D},{ }^{4}\right.$ and Navid Omidifar $\mathbb{D}^{4,5}$ \\ ${ }^{1}$ Pharmaceutical Sciences Research Center, Shiraz University of Medical Sciences, Shiraz, Iran \\ ${ }^{2}$ School of Pharmacy and Medical Sciences, University of South Australia, Adelaide SA 5000, Australia \\ ${ }^{3}$ Center for Infrastructure Engineering, Western Sydney University, Locked Bag 1797, NSW 2751, Australia \\ ${ }^{4}$ Department of Pathology, School of Medicine, Shiraz University of Medical Sciences, Shiraz, Iran \\ ${ }^{5}$ Biotechnology Research Center, Shiraz University of Medical Sciences, Shiraz, Iran \\ Correspondence should be addressed to Navid Omidifar; omidifar@gmail.com
}

Received 28 March 2020; Revised 19 June 2020; Accepted 22 June 2020; Published 3 August 2020

Academic Editor: Jianbo Yin

Copyright (c) 2020 Ahmad Gholami et al. This is an open access article distributed under the Creative Commons Attribution License, which permits unrestricted use, distribution, and reproduction in any medium, provided the original work is properly cited.

\begin{abstract}
Background. The dental pulp is a heterogeneous soft tissue that supplies nutrients and acts as a biosensor to identify pathogenic stimuli. Regeneration of the dental pulp is one of the desirable topics for researchers. Graphene oxide nanosheets (nGOs) help overexpression of the genes related to odontogenic differentiation of stem cells from dental pulps and increases attachment and proliferation of dental pulp stem cells. Despite its benefits, nGO may be considered as a threat to the environment and human health. Therefore, the purpose of this study was to evaluate the biocompatibility potential of graphene oxide (nGO), chitosan functionalized graphene oxide (nGO-CS), and carboxylated graphene (nGO-COOH) when exposed to human dental pulp stem cells (hDPSCs). Material and Methods. Some different aspects of biocompatibility of nGO, nGO-CS, and nGO-COOH were synthesized, and several intracellular effects induced by different concentrations of graphene-based nanosheets, including cell viability, intracellular oxidative damages, and various factors such as LDH, GSH, SOD, MDA, and MMP, were studied on hDPSCs. Results. According to results, $\mathrm{IC}_{50}$ was determined as $232.01,467.81$, and $\geq 1000 \mu \mathrm{g} / \mathrm{mL}$ for nGO, nGO-CS, and nGO$\mathrm{COOH}$, respectively. These results demonstrated the lower toxicity and higher cytocompatibility of nGO-CS and nGO-COOH compared to nGO. nGO-COOH not only has any adverse effect on the cell membrane and mitochondrial activity but also shows slight antioxidant activity at some concentrations. Conclusion. The findings help design safe and cytocompatible nGO derivatives for biomedical applications in dental fields.
\end{abstract}

\section{Introduction}

The dental pulp is placed in the center of teeth and is a heterogeneous soft tissue. This tissue contains various cell types and extracellular matrix molecules. As dental pulp supplies nutrients and detects the pathogenic stimuli, the vitality and freshness of dental pulp are crucial for the survival of a tooth [1]. Inflammation of the dental pulp called pulpitis is one of the most common dental diseases caused by bacterial infections and tooth decay. A pulpectomy is a traditional treatment for the irreversible pulpitis, in which the pulp tissue is removed and substituted by biocompatible materials such as gutta-percha via root canal therapy (RCT) [2]. RCT-treated teeth lost the vitality leading to fragility and vulnerability to fractures [3]. Therefore, a therapeutic strategy is required to revascularize and regenerate dental pulp [2]. Regenerative endodontics is one of the newfangled fields of regenerative medicine that its cellular and molecular aspects are yet to be discovered. Three elements are required to achieve pulp revascularization and regeneration, including dental pulp stem cells, proper scaffold, and growth factors [3]. One of the useful scaffolds is graphene derivatives that have attracted significant attention for biomedical applications due to their unique mechanical, thermal, electrical, 
optical, and biological properties, high surface-area-to-volume ratio, and unique atomic structure [4-7]. Graphenebased nanomaterials can interact with cells, supporting their adhesion, migration, proliferation, and differentiation [8, 9]. $\mathrm{nGO}$ as one the popular graphene derivatives can induce translation of some crucial intracellular protein and promote proliferation and differentiation of bone-producing cells and help bone formation [10]. The capability of nGO for bone formation was denoted by Nishida et al. in an ex vivo animal study and showed that it is fivefold more potent than the collagen scaffold [11]. Interestingly, nGO helps the expression of the genes upregulating in mineral-producing cells leading to odontogenic differentiation of stem cells from dental pulps and increases attachment and proliferation of human dental pulp stem cells (hDPSCs) [8]. Furthermore, recently, graphene oxide composites have shown the ability to differentiate hDPSCs into odontoblast-like cells and induce dentin formation via the $\mathrm{Wnt} / \beta$-catenin pathway [12].

Despite all the biomedical applications of the nanostructures, there is a need for more strategies and techniques for better understanding the effects of nGO derivatives on human health and the environment $[13,14]$. nGO may be considered as a threat due to its cytotoxicity. Thus, it is necessary to realize every aspect of its toxicity on hDPSCs before using it as a scaffold for pulp revascularization or any type of bioapplications to achieve a healthy and clean environment [15-17]. Many studies on the cytotoxicity and genotoxicity of nGO on different cell lines and animal models have confirmed its significant cytotoxicity [18-20]. Studies indicated that organs were affected by $\mathrm{nGO}$ after the migration to the human body and accumulated in tissues, resulting in the inflammation and formation of small nodules in the lungs or atherosclerotic lesions in arteries [21]. Other organs, such as reticuloendothelial systems, including the spleen, liver, and sexual organs, were triggered by $\mathrm{nGO}$ when exposed to a specific dose of nGO [22]. Researchers suggest that the functionalization of $\mathrm{nGO}$ with biocompatible molecules, such as polyethylene glycol (PEG), folic acid, and pluronic acid, can convert nGO to a nontoxic material [23-26]. Also, epoxide structures in the $\mathrm{nGO}$ family, when the ring is opened, were very reactive, which makes them more toxic; thus, converting the epoxide groups to the carboxyl group can lower the potential toxicity [27].

However, excellent studies on using $\mathrm{nGO}$ as a scaffold for tissue engineering, developing biocompatible nGO derivatives for pulp degeneration, and also a comprehensive study assessing all the factors related to cytotoxicity are still lacking. Therefore, the purpose of this study is to develop a safe and biocompatible derivative of $\mathrm{nGO}$, including $\mathrm{nGO}-\mathrm{CS}$ and nGO-COOH, and evaluate the biocompatibility potential of them in comparison to naked nGO. For this purpose, all the cytotoxicity assays including cell viability, membrane damage, cellular lactate dehydrogenase (LDH) activity, mitochondrial membrane damage and intracellular oxidative damage, and parameters related to superoxidase dismutase (SOD) and malondialdehyde (MDA) were measured to make sure about all aspects of the nGO derivative cytotoxicity on hDPSCs. According to the best knowledge of us, this work is the first study that provides a piece of comprehensive knowledge about the toxicity/safety of the graphene family on hDPSCs.

\section{Material and Methods}

2.1. Chemicals and Analytical Kits. Graphite powder, sulfuric acid $\left(\mathrm{H}_{2} \mathrm{SO}_{4}\right)$, potassium permanganate $\left(\mathrm{KMnO}_{4}\right)$, hydrogen peroxide $\left(\mathrm{H}_{2} \mathrm{O}_{2}\right), \mathrm{HCl}$ (hydrochloric acid), chloroacetic acid $\left(\mathrm{C}_{2} \mathrm{H}_{3} \mathrm{ClO}_{2}\right)$, sodium hydroxide $(\mathrm{NaOH})$, chitosan $(\mathrm{CS}), 2$ (N-morpholino)-ethanesulfonic acid )MES buffer: $\mathrm{pH} \sim 6$ ), 1-ethyl-3-(3-dimethylaminopropyl)carbodiimide (EDC), Nhydroxysuccinimide (NHS), type I collagenase, dispase II, penicillin, streptomycin, fetal bovine serum (FBS), L-glutamine, phosphate-buffered saline (PBS), sodium pyrophosphate, sodium chloride $(\mathrm{NaCl})$, Tris/hydrochloride, sodium ethylenediaminetetraacetic acid (EDTA), doxorubicin, dimethyl sulfoxide (DMSO), sodium phosphate, trichloroacetic acid (TCA), 5,5'-dithiobis-(2-nitrobenzoic acid) (DTNB), sodium carbonate $\left(\mathrm{Na}_{2} \mathrm{CO}_{3}\right)$, nitro blue tetrazolium, Triton $\mathrm{X}-100$, hydroxylamine- $\mathrm{HCl}$, and rhodamine123 were purchased from Sigma-Aldrich (St. Louis, USA).

Dulbecco's modified Eagle's medium (DMEM) was obtained from Merck Company (Germany).

The LDH cytotoxicity detection kit was purchased from Pars Azmoon company (Iran). Also, MDA and GSH assay kits were obtained from NanoAlvand company (Iran).

2.2. Preparation of $n G O$. nGOs were synthesized using Hummers' method with a slight modification [28]. Five successive stages include (a) dissolution of (1 g) graphite powder in $(23 \mathrm{~mL})$ absolute $\mathrm{H}_{2} \mathrm{SO}_{4}(v / v)$ with three days of stirring; (b) slowly pouring $(6 \mathrm{~g})$ potassium permanganate onto the solution at $4^{\circ} \mathrm{C}$; (c) heating and stirring at $40^{\circ} \mathrm{C}$ for $30 \mathrm{~min}$ and, then, stirring at a higher temperature $\left(70^{\circ} \mathrm{C}\right)$ for another $45 \mathrm{~min}$; (d) adding $6 \mathrm{~mL}$ sterile water at $105^{\circ} \mathrm{C}$ and stirring for $15 \mathrm{~min}$, again adding $40 \mathrm{~mL}$ sterile water at $100^{\circ} \mathrm{C}$ while stirring for another $15 \mathrm{~min}$; (e) adding $15 \mathrm{~mL} \mathrm{H} \mathrm{O}_{2} \mathrm{O}_{2} 35 \%$ and $150 \mathrm{~mL}$ sterile water; and (f) two consecutive centrifugation processes at $10,000 \mathrm{~g}$ for $5 \mathrm{~min}$. Finally, the precipitated substance was gathered and washed twice with $5 \% \mathrm{HCl}$ and five times with sterile water.

2.3. nGO-COOH Preparation. A modified protocol developed by $\mathrm{Yu}$ et al. was used to obtain carboxylate nGO [29]. A total of one $\mathrm{mg}$ chloroacetic acid, along with $1.2 \mathrm{mg}$ sodium hydroxide, was added to a vessel containing $\mathrm{nGO}$ suspension $20 \mu \mathrm{g} / \mathrm{mL}$. The mixture was sonicated at $80^{\circ} \mathrm{C}$ for $180 \mathrm{~min}$ until the hydroxyl and epoxy groups were converted entirely to carboxyl. Centrifugation at $13,000 \mathrm{~g}$ and washing twice were the next steps, and the resultant supernatant was redispersed in sterile water.

2.4. nGO-CS Preparation. With the presence of EDC and NHS, nGO was covalently attached to low-molecularweight chitosan (CS) via an amidation process [28]. For this, $125 \mathrm{mg}$ CS was dissolved in $25 \mathrm{~mL}$ MES buffer ( $\mathrm{pH} \sim 6$ ) along with $100 \mathrm{mg} \mathrm{nGO}$ and sonicated at room temperature for 60 min. Under $\mathrm{N}_{2}$ gas, $300 \mathrm{mg}$ EDC and $400 \mathrm{mg}$ NHS were carefully poured into the reaction vessel, stirred for $20 \mathrm{~min}$, and sonicated for $300 \mathrm{~min}$. In the final step, the product 

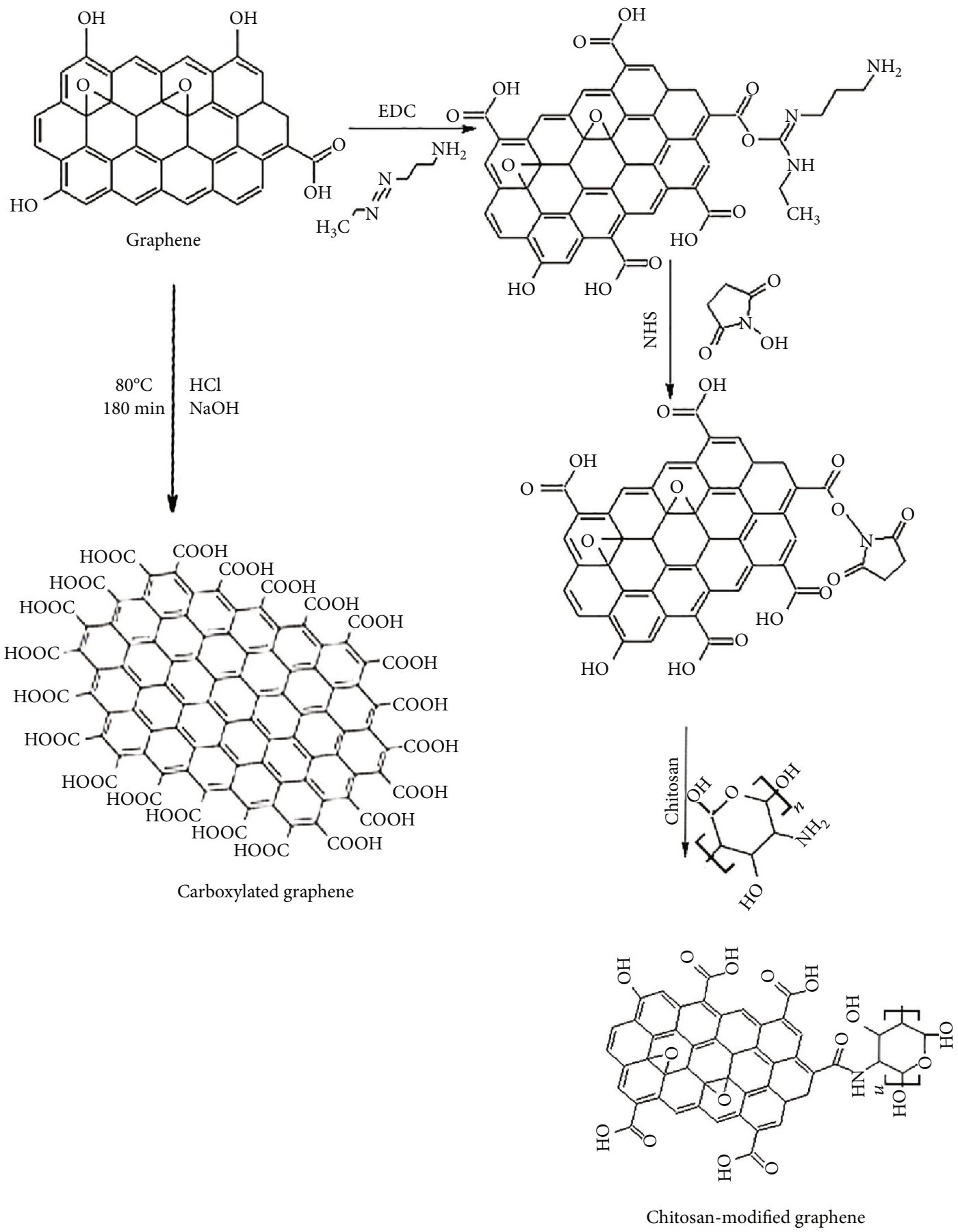

FIgURE 1: The synthesis pathway of used nanostructures.

was stirred overnight and filtered by a 0.2 -micron microporous membrane. In order to remove remnant CS, the product was subsequently washed with acetic acid $0.1 \mathrm{M}$ and dialyzed in $10 \mathrm{kDa}$ cut-off dialysis bag for four days. Figure 1 illustrated schematically the synthesis pathway of nanostructures used in this study.

2.5. SEM, TEM, UV-Vis, and FT-IR Characterizations of Nanosheets. A Fourier transform infrared (FT-IR) spectrometer (Perkin Elmer, UK) was used with $\mathrm{KBr}$ pellet to record the FT-IR spectra. The UV-Vis spectra were recorded using a T80+ UV-Vis spectrometer (PG Instruments, Australia). The Transmission Electron Microscopy (TEM) micrographs were obtained using a Zeiss EM900 (Carl Zeiss, Germany) microscope operating at $80 \mathrm{kV}$ on formvar/carbon-coated copper grids. A scanning electron microscopy (SEM) VEGA2-LMU microscope (Tescan, Czech) was utilized for SEM characterizations along with energy-dispersive X-ray spectroscopy (EDS) which has been used to determine combinations of samples. X-ray diffraction (XRD: Bruker D8, advance, $\mathrm{k} \alpha: \mathrm{Cu}$ ) was used to examine crystalizing material nanostructures. 
2.6. Cell Culture Process. After informed consent, hDPSCs were extracted from the molar tooth of 40-year-old adult subjects. After isolating cells from dental pulp, they were harvested and soaked in a solution containing type I collagenase $(3 \mu \mathrm{g} / \mu \mathrm{L})$ and dispase II $(4 \mu \mathrm{g} / \mu \mathrm{L})$ in DMEM incubated at $37^{\circ} \mathrm{C}$ for about one $h$. Then, to get a suspension of cells, the solution was filtered through Falcon Cell Strainers $(100 \mu \mathrm{m})$. The mixture was incubated at $37^{\circ} \mathrm{C}$, in flasks, and cultivated in medium containing DMEM with penicillin $(100 \mathrm{IU} / \mathrm{mL})$, streptomycin $(100 \mathrm{mg} / \mathrm{L})$, fetal bovine serum (FBS, $10 \% \mathrm{v} / \mathrm{v}$ ), L-glutamine $(2 \mathrm{mM})$, and $5 \% \mathrm{CO}_{2}$. After 72 hours, reached cells were consequently trypsinized, appended, and overlaid at clonal bulk (1.6 cells $/ \mathrm{mL})$. On the $7^{\text {th }}$ day, four colonies were selected. Afterward, hDPSCs were extended to get $85 \%$ confluency and around $4 \times 10^{6}$ cells, which have been used for the experimental study.

2.7. Cell Exposure to Compounds. All biological assays, including cell viability, intracellular oxidative damage, and membrane mitochondrial potential assessments, needed exposure of cells to all experiment compounds. In order to achieve sufficient cell numbers, specific concentrations of grapheme family nanosheets were added to cells in a 12-well cell culture plate. After $24 \mathrm{~h}$, the cells were detached using trypsin/EDTA and then washed at $4^{\circ} \mathrm{C}$ with phosphatebuffered saline (PBS) three times. Then, the cells were centrifuged at $4000 \mathrm{rpm}$ for $10 \mathrm{~min}$. A cocktail of cell lysis buffer including Triton X-100 (1\%), sodium pyrophosphate $(2.5 \mathrm{mM})$, sodium chloride $(150 \mathrm{mM})$, Tris/hydrochloride $(20 \mathrm{mM})$, and sodium EDTA $(1 \mathrm{mM})$ was supplemented to the cell biomass and then centrifuged at $13,000 \mathrm{rpm}$ for $10 \mathrm{~min}$ at $4^{\circ} \mathrm{C}$. Finally, the biomass was discarded and solution, which contains all components of the cell, but the cell wall was kept at $-20^{\circ} \mathrm{C}$ until the following biological assays.

2.8. Cell Viability Assessment. In vitro cytotoxicity investigation was conducted using MTT assays [30, 31]. The hDPSCs were seeded in 96-well plates at a density of 10,000 cells/well in $100 \mu \mathrm{L}$ DMEM. After overnight incubation at $37^{\circ} \mathrm{C}$ in humidified 5\% $\mathrm{CO}_{2}$-containing atmosphere, the medium was added to the 96-well plates with doxorubicin (as a standard cytotoxic agent), nGO, nGO-COOH, and nGOCS at six different concentrations $(25,50,100,250,500$, and $1000 \mu \mathrm{g} / \mathrm{mL}$ ); the cells were cultured for $24 \mathrm{~h}$. After the extraction, $25 \mu \mathrm{L}$ MTT solution was added, and the cells continued to be incubated for an additional four h. Finally, the medium was removed, and $100 \mu \mathrm{L}$ DMSO was added to 96-well plates and thoroughly shaken for $15 \mathrm{~min}$. The absorption strength of each well was recorded at $570 \mathrm{~nm}$ by using a microplate reader. The relative cell viability was measured by comparing the tested wells (containing cells and nanostructures) with the control wells (containing cells). The cell viability was measured from

$$
\text { cell viability }(\%)=\frac{\mathrm{OD}_{\mathrm{s}}-\mathrm{OD}_{\mathrm{b}}}{\mathrm{OD}_{\mathrm{c}}-\mathrm{OD}_{\mathrm{b}}} \times 100 \text {, }
$$

where $\mathrm{OD}_{\mathrm{s}}, \mathrm{OD}_{c}$, and $\mathrm{OD}_{\mathrm{b}}$ are optical density values of sample wells, positive control, and background wells, respec- tively. $\mathrm{IC}_{50}$ was defined as the concentration leading to $50 \%$ inhibition of cell viability.

2.9. LDH Assay. Cell membrane damage that occurred with the nanomaterials was assessed using an LDH cytotoxicity detection kit, according to Zamani et al. [32]. In this test, the release of cytoplasm enzyme lactate dehydrogenase (LDH) was spectrophotometrically measured using a microplate reader. The hDPSCs were cultured in 96-well cell culture plates and treated with different concentrations of the graphene-based nanomaterials $(25,50,100,250,500$, and $1000 \mu \mathrm{g} / \mathrm{mL}$ ) for $24 \mathrm{~h}$. Then, the supernatant was transferred to vials, which are incubated with the reaction mixture. Absorbance was read at $340 \mathrm{~nm}$, and the activity of lactate dehydrogenase enzyme was showed in units per $\mathrm{mL}$ using

$$
\begin{aligned}
\text { Volume activity }(\mathrm{U} / \mathrm{mL}): & {\left[\frac{\mathrm{OD}_{340} *\left(V_{\text {reaction }}+V_{\text {enzyme }}\right)}{6.22 * V_{\text {enzyme }}}\right] } \\
& * \text { dilution factor. }
\end{aligned}
$$

2.10. Lipid Peroxide (LPO) Level. The level of LPO was defined by calculating the MDA made using Nalondi ${ }^{\mathrm{TM}}$ Lipid Peroxidation Assay Kit-MDA. According to the instruction of the kit, $100 \mu \mathrm{L}$ cell lysate was combined with $1.9 \mathrm{~mL}$ sodium phosphate buffer $(0.1 \mathrm{M}, \mathrm{pH} 7.4)$ and incubated at $37^{\circ} \mathrm{C}$ for $60 \mathrm{~min}$. Then, before centrifugation $(13,000 \mathrm{rpm}$ for $10 \mathrm{~min})$ at room temperature, 5\% TCA was supplemented. The supernatant was blended with thiobarbituric acid $(1 \%, 1 \mathrm{~mL})$ and set at $100^{\circ} \mathrm{C}$ in a water bath for about half of an hour. The OD of the cooled suspension was observed at $532 \mathrm{~nm}$ and converted to MDA (percent compared to the control).

2.11. GSH Level. NarGul ${ }^{\mathrm{TM}}$-Glutathione assay kit-GSH was used to determine the level of GSH. A total of $100 \mu \mathrm{L}$ of the cell lysate was added to TCA $(5 \%, 900 \mu \mathrm{L})$ then centrifuged $(13,000 \mathrm{rpm})$ for $10 \mathrm{~min}$ at a cold temperature $\left(4^{\circ} \mathrm{C}\right)$. $500 \mu \mathrm{L}$ supernatant was mixed with $0.01 \%, 1.5 \mathrm{~mL}$ Ellman's reagent (DTNB), and detected at $412 \mathrm{~nm}$ using a UVSpectrophotometer. The GSH amount was declared in terms of percentage after comparing it with the control.

2.12. SOD Level. The Nasdox ${ }^{\mathrm{TM}}$ superoxide dismutase (SOD) activity assay kit was used to measure the effect of nanomaterials on intracellular SOD levels. After exposure to different concentrations of graphene-based nanomaterials $(0,25,50$, $100,250,500$, and $1000 \mu \mathrm{g} / \mathrm{mL}$ ), the cells were harvested and lysed in lysis buffer at $4^{\circ} \mathrm{C}$. A total of $2.1 \mathrm{~mL}$ of the mixture contained $1.9 \mathrm{~mL}$ sodium carbonate buffer $(50 \mathrm{mM})$, $30 \mu \mathrm{L}$ nitro blue tetrazolium $(1.6 \mathrm{mM}), 6 \mu \mathrm{L}$ Triton $\mathrm{X}-100$ $(10 \%)$, and $20 \mu \mathrm{L}$ hydroxylamine- $\mathrm{HCl}(100 \mathrm{mM})$. Consequently, absorbance at $560 \mathrm{~nm}$ for $5 \mathrm{~min}$ was measured.

2.13. MMP. MMP was calculated using a lipophilic cationic dye, rhodamine-123. In brief, the hDPSCs were cultured in a 12-well cell culture plate and incubated with nanomaterials at $37^{\circ} \mathrm{C}$ for $24 \mathrm{~h}$, and then, the nanomaterials were removed, 


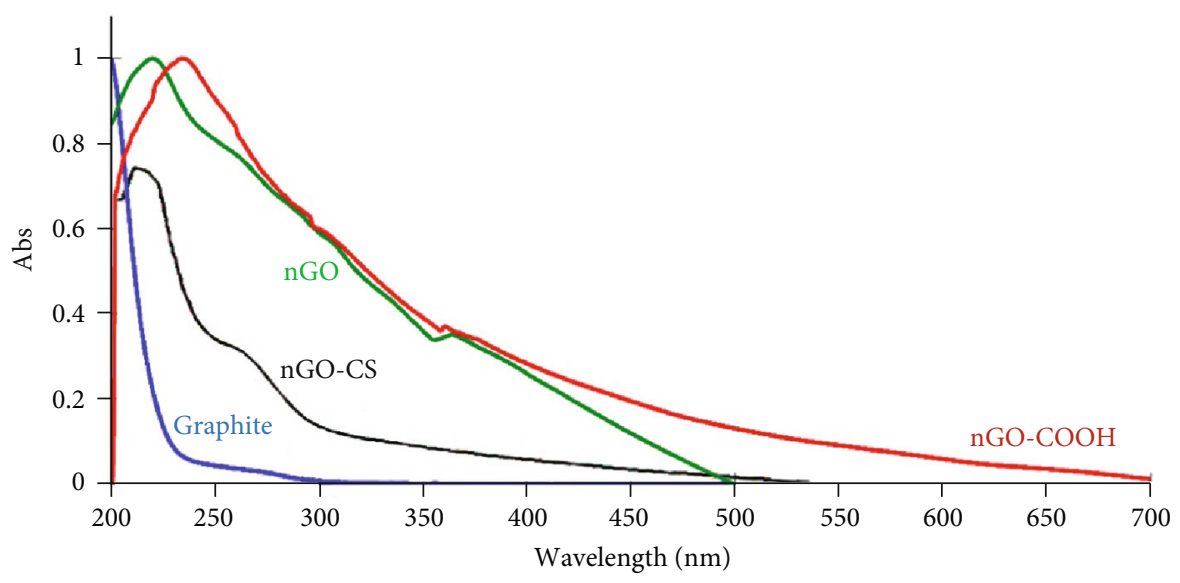

FIGURe 2: UV-Vis spectra of graphite, nGO, nGO-CS, and nGO-COOH.

and rhodamine-123 $(2 \mu \mathrm{M})$ was added to the cells and incubated for $30 \mathrm{~min}$ at $37^{\circ} \mathrm{C}$. Rhodamine-123, a fluorescent molecule, enters the mitochondrial matrix; therefore, fluorescence emission is an index for mitochondrial transmembrane potential, and it was quantitatively measured by a fluorescence microplate reader (CLARIOstar, Germany) at the wavelength of $480 \mathrm{~nm}$. The MMP percent for untreated cells (control) was considered as $100 \%$, and the other samples were calculated in comparison with the control.

2.14. Statistical Analysis. The data were illustrated as the mean \pm standard deviation. Statistical difference was analyzed using the one-way analysis of variance (ANOVA) followed by the Tukey post hoc test. $p$ value $<0.05$ was considered significant.

\section{Results}

3.1. Characterization of Graphene Nanosheets. According to Hummer's method, graphite was peroxidized by $\mathrm{H}_{2} \mathrm{SO}_{4}$ and $\mathrm{KMnO}_{4}$ to maintain oxygen-containing groups (such as carboxylic acid, epoxy, and hydroxyl) on the surface of nGO [33]. The extra amount of $\mathrm{KMnO}_{4}$ was used to ascertain the oxidation. In the presence of $\mathrm{N}$-(3-dimethylaminopropyl)- $\mathrm{N}^{\prime}$-ethyl carbodiimide hydrochloride (EDC) and $\mathrm{N}$ hydroxysuccinimide (NHS) in 4-morpholineethanesulfonic acid (MES buffer), chitosan molecules were attached to nGO via amide covalent bonds. EDC activated the carboxyl groups on nGO to form an active ester, which was stabilized further by NHS. The active ester tends to become an amide linkage between nGO and low molecular CS. nGO-COOH was synthesized in an alkaline condition $(\mathrm{pH}>9)$ in which nGO was activated in the presence of chloroacetic acid; all nGO hydroxyl groups were converted to carboxylic acid moieties. Figure 2 shows the UV-Vis spectra of graphite, nGO, nGO-COOH, and nGO-CS. After the dissolution of samples in sterile distilled water, the UV-Vis apparatus was set to a range of 200-700 $\mathrm{nm}$. The characteristic peak of $\pi-\pi *$ transition of $\mathrm{C}=\mathrm{C}$ bonds at $230-235 \mathrm{~nm}$ wavelength is seen in nGO, nGO-COOH, and nGO-CS while the graphite spectra lack such a peak. A small shoulder in the range of 300-
$375 \mathrm{~nm}$ is contributed to the $\pi-\pi *$ transition of $\mathrm{C}=\mathrm{O}$, which is shifted to $<300 \mathrm{~nm}$ after coupling nGO with CS.

The FT-IR analysis of nGO, nGO-COOH, and nGO-CS is verified upon a wide and strong peak at around $3420 \mathrm{~cm}^{-1}$ (Figure 3). For nGO, nGO-COOH, and nGO$\mathrm{CS}$, this peak is correlated with the absorption of $\mathrm{O}-\mathrm{H}$ stretching bonds; the graphite spectrum does not have such a peak. For nGO, a relatively small peak is seen at around $2920 \mathrm{~cm}^{-1}$, which is related to $\mathrm{C}-\mathrm{H}$ stretching bond, and at around 1050 to $1250 \mathrm{~cm}^{-1}$ corresponding to $\mathrm{O}-\mathrm{H}$ primary bonds. Concisely, the analysis of peaks confirms the presence of oxygen-containing groups in nGO and the absence of that in graphite. There is another characteristic peak for $\mathrm{nGO}$ and $\mathrm{nGO}-\mathrm{COOH}$ at around $1700 \mathrm{~cm}^{-1}$, which corresponded to $\mathrm{C}=\mathrm{O}$ bonds in carboxyl groups. As shown in Figure 3, this peak in $\mathrm{nGO}-\mathrm{COOH}$ is sharper than the one for $\mathrm{nGO}$, indicating that the number of carboxylic acid groups increased. It seems that these groups originated from the treatment of nGO with chloroacetic acid. Comparing with nGO and nGO-COOH, the peak disappeared in the nGO-CS spectra, which demonstrates that carboxyl groups of nGO react with amine groups of CS. The FT-IR spectrum of nGO-CS shows a peak at $1640 \mathrm{~cm}^{-1}$, which contributed to the stretching vibrations of $\mathrm{NHCO}$ at $1640 \mathrm{~cm}^{-1}$ due to the amide linkage between amine groups of CS chains and carboxyl groups of nGO. Because of the interaction between hydroxyl groups of nGO and functional groups of CS, the intensity of the peak of C-O stretch for nGO-CS (at $1074 \mathrm{~cm}^{-1}$ ) is higher than the ones for $\mathrm{nGO}$ (at $\left.1056 \mathrm{~cm}^{-1}\right)$ and $\mathrm{nGO}-\mathrm{COOH}\left(1079 \mathrm{~cm}^{-1}\right)$. In short, the strong presence of $-\mathrm{COOH}$ bonds in $\mathrm{nGO}$ $\mathrm{COOH}$ and the attachment of CS to nGO are confirmed.

Figure 4 presents the TEM and SEM morphology images of nGO, nGO-COOH, and nGO-CS nanosheets. The SEM micrographs of $\mathrm{nGO}$ (Figure 4, A1) and $\mathrm{nGO}-\mathrm{COOH}$ (Figure 4, B1) demonstrate a similar structure for nGO before and after carboxylation. Also, the EDS analysis of $\mathrm{nGO}$, nGO-CS, and nGO-COOH has been summarized in Table 1. Both nGO and nGO-COOH have loosely stacked and typical wrinkled sheet structure. The wrinkled nature is essential to prevent the collapse-back phenomenon to graphitic form. There are some nanopores on the surface of nGO-COOH, maybe due to partial destruction of nGO 


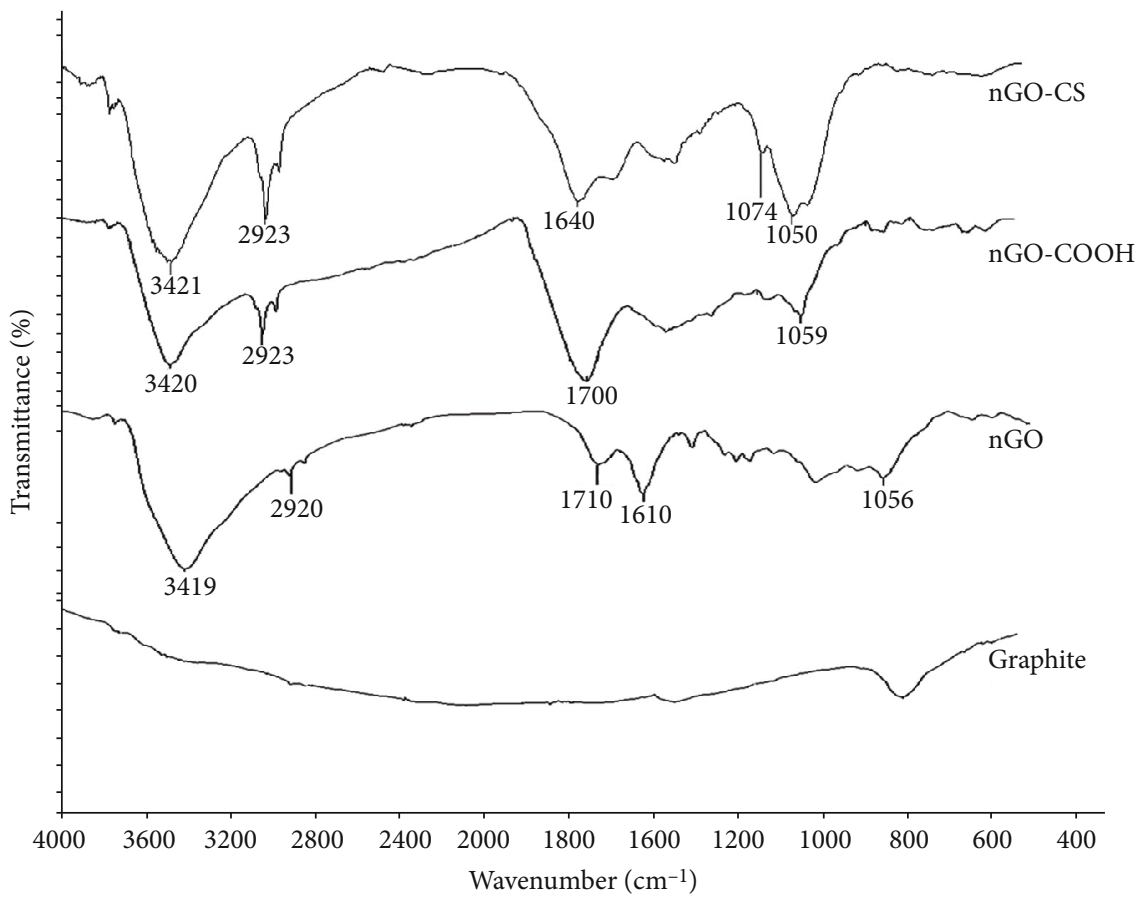

Figure 3: FT-IR spectra of graphite, nGO, nGO-CS, and nGO-COOH for the wavelength range of $600-4000 \mathrm{~cm}^{-1}$. The peak at $1700 \mathrm{~cm}^{-1}$ is the characteristic peak for $\mathrm{nGO}$ and $\mathrm{nGO}-\mathrm{COOH}$; the peak for $\mathrm{nGO}-\mathrm{COOH}$ is sharper than that of nGO.
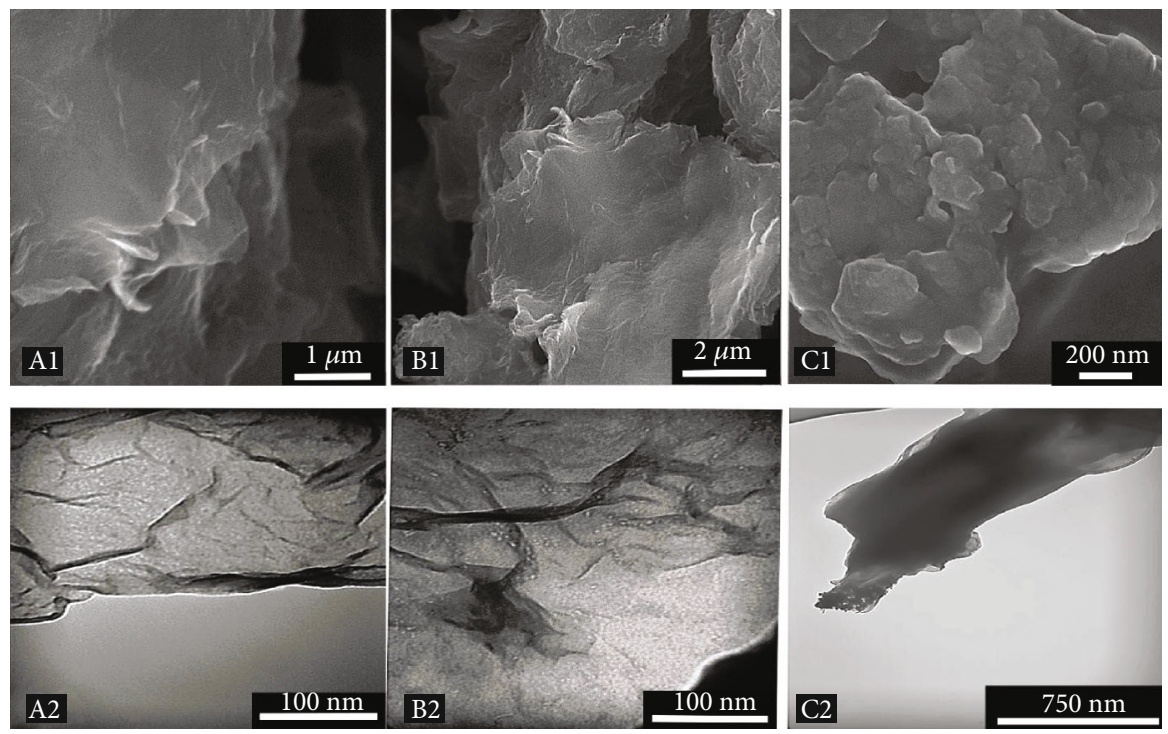

FIGURE 4: SEM images of (A1) nGO and (B1) nGO-COOH with wrinkled sheet structure and smooth surface and (C1) nGO-CS with a rough surface. TEM images of (A2) nGO and (B2) nGO-COOH with thin sheet structure and (C2) nGO-CS dense and thick structure.

TABLE 1: EDS analysis of the nGO, nGO-CS, and nGO-COOH.

\begin{tabular}{lccc}
\hline Element & nGO (Wt\%) & nGO-CS (Wt\%) & nGO-COOH (Wt\%) \\
\hline $\mathrm{C}$ & 59.11 & 36.36 & 66.78 \\
$\mathrm{O}$ & 37.31 & 60.07 & 31.67 \\
$\mathrm{~N}$ & 0 & 2.42 & 0 \\
$\mathrm{~S}$ & 3.54 & 1.15 & 1.34 \\
\hline
\end{tabular}

nanosheets during the process of carboxylation. In the TEM images, the wrinkled surface is observed in nGO (Figure 4, A2) and nGO-COOH (Figure 4, B2), along with the nanopores on nGO-COOH. Compared to $\mathrm{nGO}$, nGO-CS has a thickened and rough structure where chitosan covers the surface of nGO (Figure 4, C2). The morphological difference between nGO, nGO-COOH, and nGO-CS and larger and smoother surface in nGO-CS (Figure 4, C1) corresponds to amide and hydrogen linkage between functional groups of nGO and CS. 


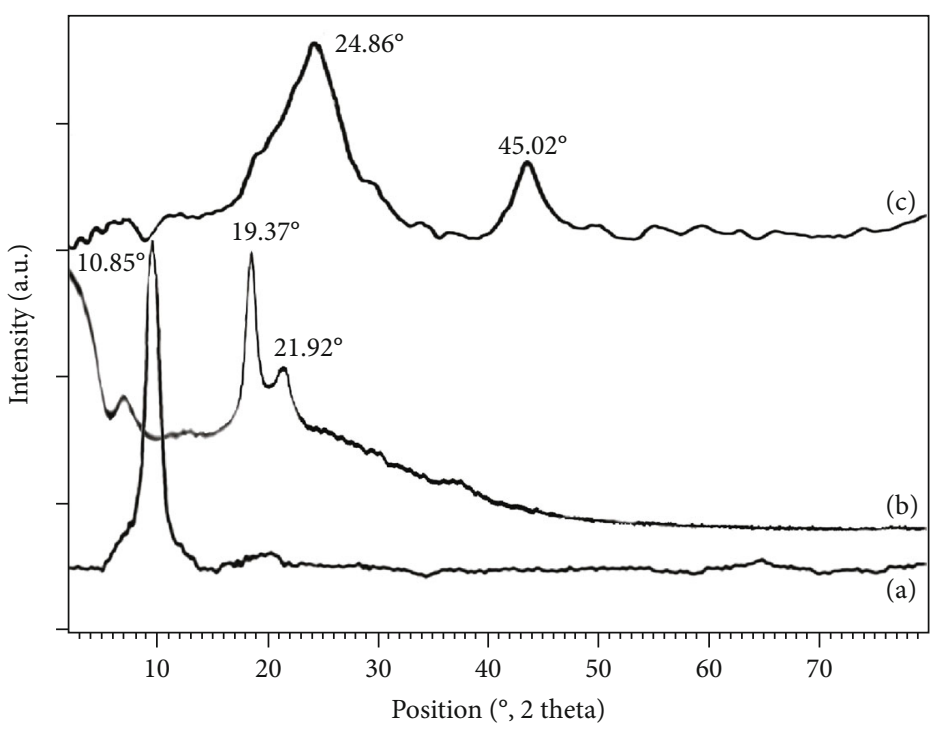

Figure 5: XRD pattern of (a) nGO, (b) nGO-CS, and (c) nGO-COOH.

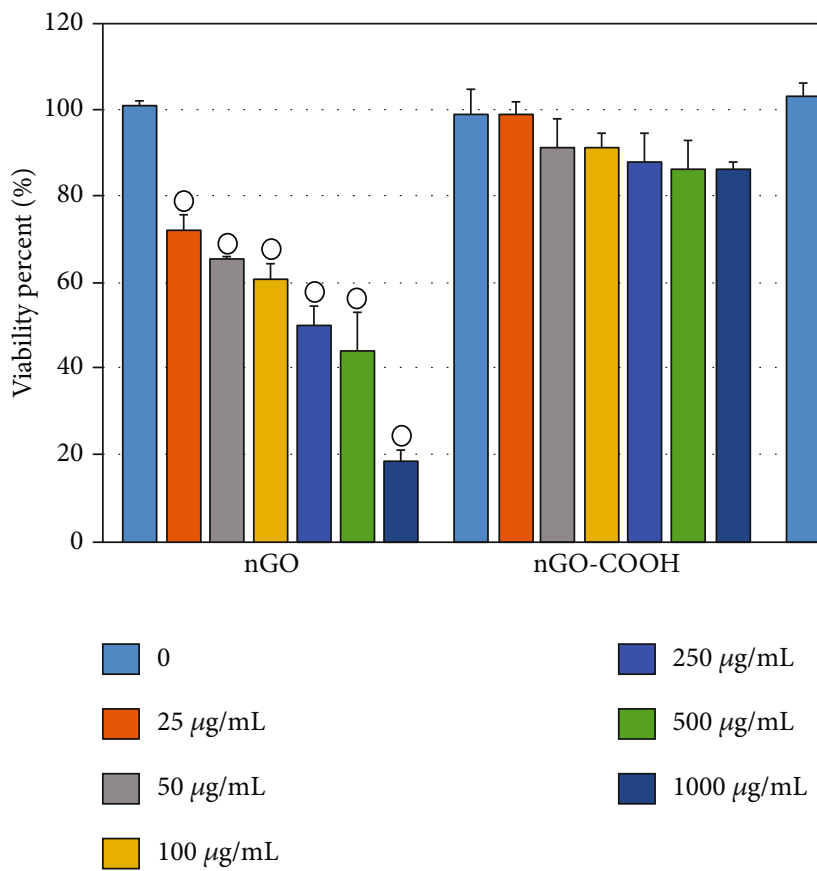

FIGURE 6: Viability of hDPSCs exposed to graphene-based nanomaterials at the concentrations of $25,50,100,250,500$, and $1000 \mu \mathrm{g} / \mathrm{mL}$ determined by the MTT assay after 24 hours. The results are given in percent compared to the untreated control group. Results are the mean \pm SEM (bars) of three independent experiments each carried out in triplicate. ${ }^{*} p$ value $<0.05$.

The XRD pattern of nGO is also displayed in Figure 5(a). A sharp peak was observed at approximately $10.85^{\circ}$, indicating the crystal plane of exfoliated nGO. nGO-CS showed a broad peak at $2 \theta=19.37^{\circ}, 21.92^{\circ}$ (Figure 5(b)), exhibiting an amorphous structure and physical and noncovalent interactions such as hydrogen bond between chitosan and nGO. Also, the diffraction intensity of nGO-CS composites at $2 \theta$ $=18.71^{\circ}$ showed an increase in the crystallinity of CS after adding loading on nGO. The XRD patterns for GO-COOH (Figure 5(c)) exhibit the main peaks at 24.86 and 45.02 .
3.2. Cell Viability Assays. The cytocompatibility of nGO and its functionalized derivatives at the concentrations of 25,50 , $100,250,500$, and $1000 \mu \mathrm{g} / \mathrm{mL}$ were evaluated on hDPSCs using the MTT assay and LDH assay. As shown in Figure 6, the cytotoxicity of nGO, nGO-CS, and nGO-COOH is dose-dependently increased. At each concentration, the cell viability of the $\mathrm{nGO}-\mathrm{COOH}$ group was significantly more than that of the $\mathrm{nGO}$ and nGO-CS groups. Even at $1000 \mu \mathrm{g} / \mathrm{mL}$ of $\mathrm{nGO}-\mathrm{COOH}$, the cell viability was about $90 \%$, obviously lower toxicity than others. On the contrary, 
TABLE 2: $\mathrm{IC}_{50}$ values of graphene oxide nanosheets corresponding to human dental pulp stem cells.

\begin{tabular}{lccc}
\hline & nGO & nGO-COOH & nGO-CS \\
\hline $\mathrm{IC}_{50}(\mu \mathrm{g} / \mathrm{mL})$ & 232.01 & $>1000$ & 467.81 \\
\hline
\end{tabular}

the cell viability of nGO at the concentration of $1000 \mu \mathrm{g} / \mathrm{mL}$ is lower than $20 \%$, which is considered as potentially cytotoxic. According to MTT results, the survival curves were drowned, and $\mathrm{IC}_{50}$ of components were showed in Table 2, and the results showed that the highest biocompatible nanosheet was nGO-COOH ( $\mathrm{IC}_{50}$ above $1000 \mu \mathrm{g} / \mathrm{mL}$ ).

The LDH activity assay was also done to evaluate the effect of graphene nanosheets on the cell membrane integrity of hDPSCs. The results demonstrated that nGO exposure caused membrane damage, and LDH leakage was increased in a dose-dependent manner (Figure 7). Although exposure to nGO-CS leads to lower $\mathrm{LDH}$ leakage than $\mathrm{nGO}$, nGOCOOH had no significant effect on LDH leakage in hDPSCs. The results obtained from cytotoxicity assessments showed that some types of graphene nanosheets affect their biocompatibility (Figures 6 and 7). nGO without any functionalization, at the examined concentrations, did not show sufficient biocompatibility for biomedical applications, and, on the contrary, nGO-COOH did not induce cytotoxicity to hDPSCs.

3.3. Intracellular Oxidative Damage. Increasing oxidative processes in the cells, along with inhibition of mitochondrial activity and increased membrane permeability, is another important pathway for nanomaterial cytotoxicity. Glutathione (GSH) is an antioxidant molecule that protects the cell and intracellular organelles against damage caused by oxidative stress and plays a crucial role in establishing cellular redox homeostasis. Therefore, the concentration of GSH straightly represents the antioxidant level of the tissue. Exposure to nGO and their functionalized derivatives at the concentrations of $25,50,100,250,500$, and $1000 \mu \mathrm{g} / \mathrm{mL}$ for sixh affects intracellular GSH levels in a dose-dependent manner (Figure 8). There was a significant decrease in the level of GSH in the nGO group compared to the control ( $p$ value $<0.05$ ). In contrast, cell exposure to nGO-COOH did not lead to GSH depletion, and CS-nGO had an intermediate effect. These results revealed that the oxidative stress level was elevated in the nGO-treated cells.

In addition to the GSH level, we examined some other parameters which are correlated with oxidative stress, such as the superoxide dismutase (SOD) activity as well as malondialdehyde (MDA). SOD is an enzyme which is considered as a primary antioxidant mechanism of the cell. It converted $\mathrm{O}_{2}$ radicals into $\mathrm{H}_{2} \mathrm{O}_{2}$. Therefore, the antioxidant ability of materials was correlated with SOD activity in the cells. In this study, the effect of nGO and its functionalized derivatives was investigated on SOD activity using a commercial colorimetric assay kit (Navand Salamat, Iran). The results showed that nGO significantly lowered the SOD activity compared to the control (Figure 9) ( $p$ value $<0.05$ ). The lowest decrease in SOD activity has been seen in the cell, which is exposed to nGO-COOH. Interestingly, $100 \mu \mathrm{g} / \mathrm{mL}$ of nGO-COOH sig- nificantly elevated SOD activity in the cells in comparison with the control group, while, at this concentration of naked nGO, we observed a severe decrease in superoxide dismutase activity. Although nGO-CS displayed a lower reduction in superoxide dismutase activity compared to $\mathrm{nGO}$, it still caused a significant decrease compared to the control group, especially at high concentrations.

MDA level measurement was applied to elucidate the lipid membrane peroxidation induced by the graphene family. A concentration-dependent pattern was also observed in increasing the MDA level of cells after $24 \mathrm{~h}$ exposure to $\mathrm{nGO}$ and nGO-CS (Figure 10). However, nGO-COOH did not significantly increase the intracellular MDA concentration at any concentration compared to the control group ( $p$ value $>0.05)$. The nGO-treated group at 500 and $1000 \mu \mathrm{g} / \mathrm{mL}$ resulted in the highest MDA concentration, approximately twice the nGO-COOH level group.

3.4. Mitochondrial Membrane Potential (MMP). Since we intended to conduct a comprehensive study on the cytotoxicity of the nGO family to human dental stem cells, mitochondrial function was further explored under graphene exposure. The loss of MMP was investigated using rhodamine-123, which revealed a dose-dependent decrease in the MMP following exposure to graphene nanosheets (Figure 11). At a high concentration $(1000 \mu \mathrm{g} / \mathrm{mL})$ of $\mathrm{nGO}$, MMP was reduced to $12.3 \%$ of the control group in the hDPSCs. nGO disturbed the MMP of hDPSCs significantly more than the control group, and nGO-COOH did not impair it even at high concentrations (85.59\% of the control group). In comparison, nGO-CS disrupted the MMP only at high concentrations ( $47.27 \%$ of the control group).

\section{Discussion}

Exceptional mechanical strength, electrical conductivity, and thermal stability of nGO derivatives provided the high potential for tissue engineering in dental fields and regenerative dentistry. The critical point to the exciting future of graphene and its related nanomaterials in the dental field is that graphene can induce osteogenic stem cell differentiation. However, there is an increasing concern about their adverse biological effects and toxicity not only to humans but also to the environment $[22,34,35]$. Liao et al. reported that the high toxicity of graphene and graphene oxide attributes to the type of media and interaction with cells [34]. Although Chang et al. reported nGO toxicity only at higher concentrations $(1000 \mu \mathrm{g} / \mathrm{mL})$, many other studies revealed much lower biocompatibility [36]. As a solution, several attempts have focused on the functionalization of the nGO family to reduce their cytotoxicity. For example, functionalization by polyethyleneimine (PEI) increased their cytocompatibility on the human epithelial carcinoma cell line, even at concentrations higher than $300 \mu \mathrm{g} / \mathrm{mL}$ [37]. In the present study, nGO-COOH and nGO-CS are demonstrated with a high cytocompatibility than $\mathrm{nGO}$ with $\mathrm{IC}_{50} \geq 1000,500$, and $250 \mu \mathrm{g} / \mathrm{mL}$, respectively. It is well known that plasma membrane damage and induction of oxidative stress play a crucial role in the toxicity property of nGO $[38,39]$. However, some 


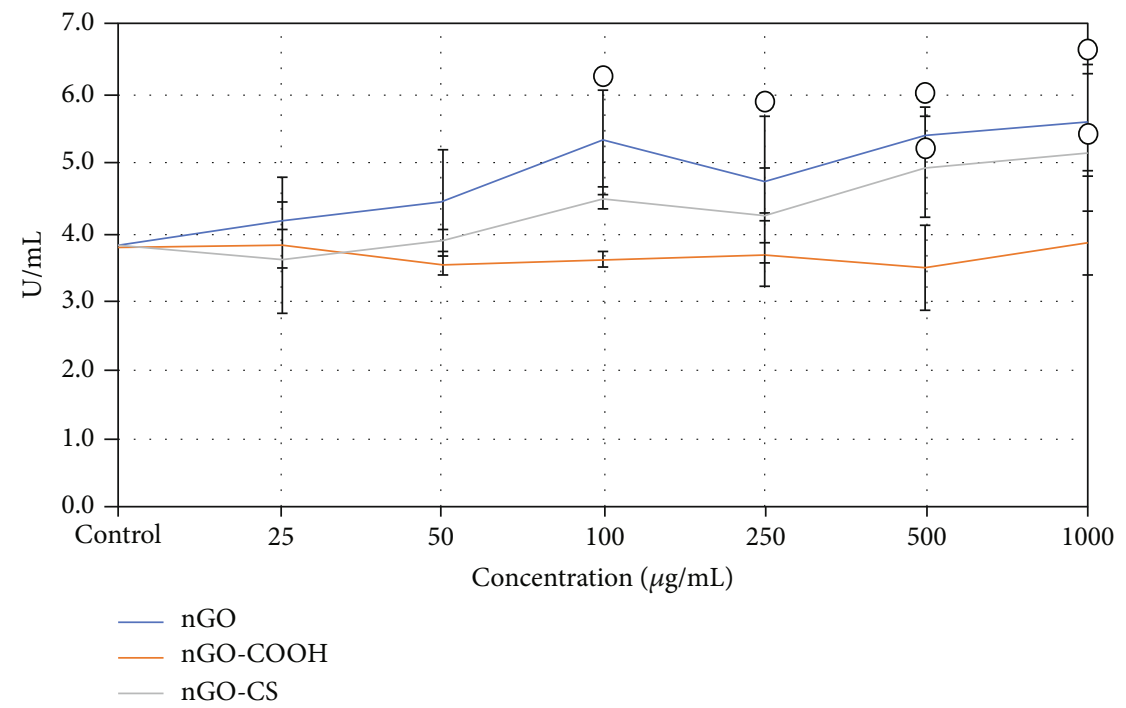

FIGURE 7: LDH leakage from hDPSCs after incubation with different concentrations of the graphene-based nanomaterials (25, 50, 100, 250, 500 , and $1000 \mu \mathrm{g} / \mathrm{mL}$ ) for $24 \mathrm{~h}$. The $\mathrm{LDH}$ results are given in the activity unit/mL. Results are the mean \pm SEM (bars) of three independent experiments each carried out in triplicate. Although exposure to nGO-CS leads to lower LDH leakage than nGO, nGO-COOH had no significant effect on LDH leakage in hDPSCs. ${ }^{*} p$ value $<0.05$.

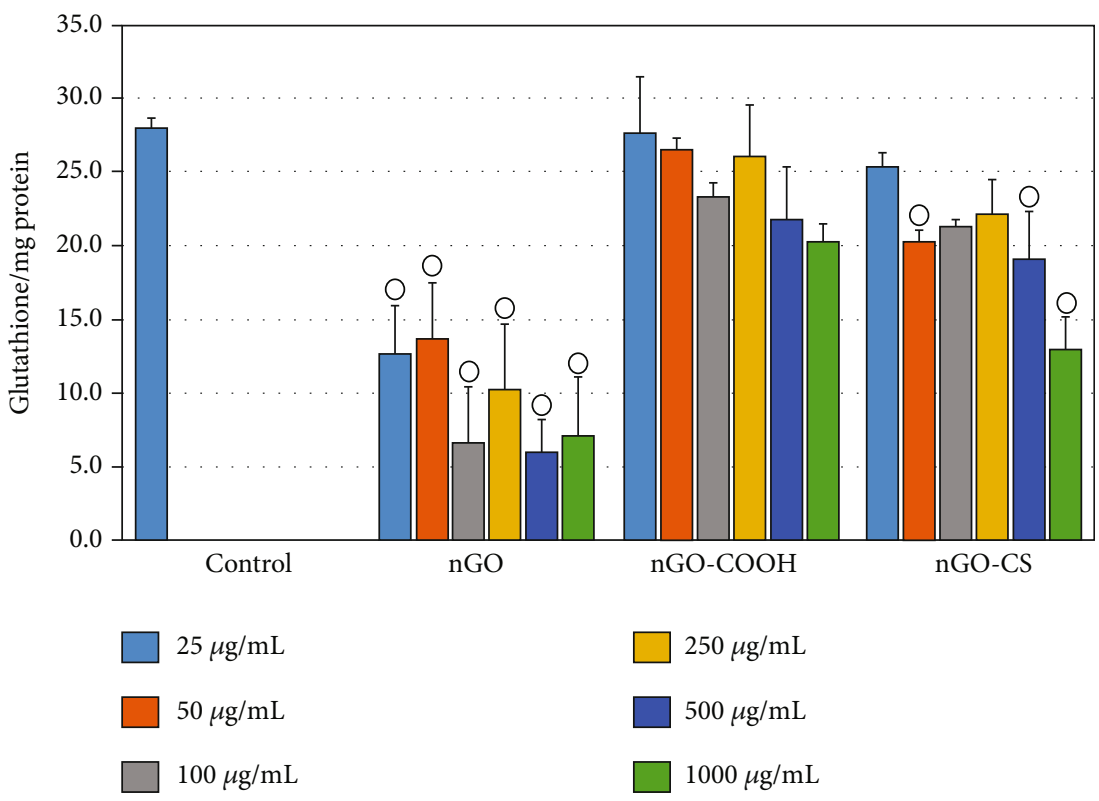

FIGURE 8: GSH level in the hDPSCs after $24 \mathrm{~h}$ incubation with different concentrations of graphene-based nanomaterials (25, 50, 100, 250, 500 , and $1000 \mu \mathrm{g} / \mathrm{mL}$ ). The GSH results are given in glutathione/mg protein. Results are the mean \pm SEM (bars) of three independent experiments each carried out in triplicate. There was a significant decrease in the level of GSH in the nGO group compared to the control ( $p$ value $<0.05)$. In contrast, cell exposure to $n G O-C O O H$ did not lead to GSH depletion, and CS-nGO had an intermediate effect. ${ }^{*} p$ value $<0.05$.

studies showed that nGO could not easily damage the cell membrane after penetrating various cells $[40,41]$. This controversy may be related to different modes of synthesis, dispersants, and cell lines. It seems that the dominant mechanism of nGO cytotoxicity is oxidative stress and damages to crucial organelles such as mitochondria and membranes. It is expected that changing nGO's functional group to more soluble and biocompatible groups can alter their cytocompatibility [36].
In our study, the epoxide and ester groups of nGO were activated by the dissociation of $\mathrm{H}^{+}$and nucleophilic substitution reaction of oxygen ions in an alkaline environment. In the final stage, the epoxide and ester groups were converted to carboxyl moieties. nGO sheets were decorated with many hydroxyl groups at basal planes and carbonyl groups at the edges, which inherited more solubility and low toxicity [42]. Besides, the hydrogen bond between water (the most dominant biologic solvent) and the carboxyl group was more 


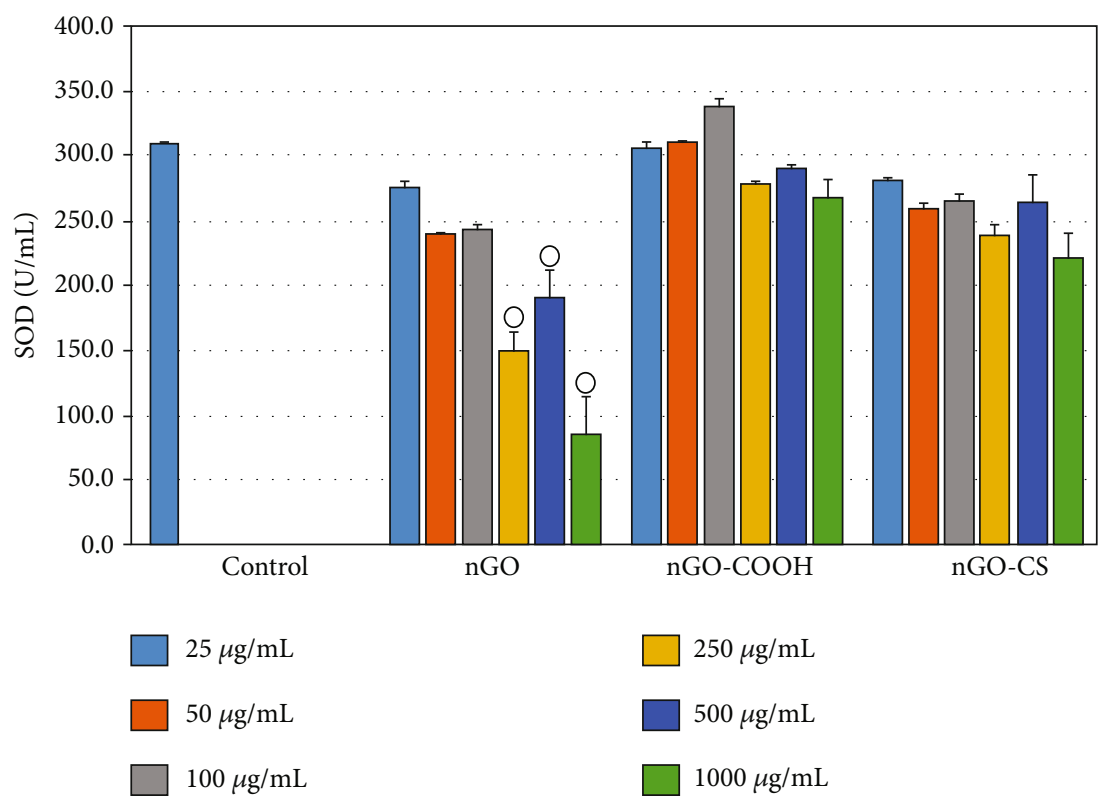

FIGURE 9: SOD in the hDPSCs after $24 \mathrm{~h}$ incubation with different concentrations of graphene-based nanomaterials $(25,50,100,250,500$, and $1000 \mu \mathrm{g} / \mathrm{mL}$ ). The SOD results are given in the activity unit $/ \mathrm{mL}$. Results are the mean \pm SEM (bars) of three independent experiments each carried out in triplicate. The results showed that nGO significantly lowered the SOD activity compared to the control $(p$ value $<0.05) .{ }^{*} p$ value $<0.05$.

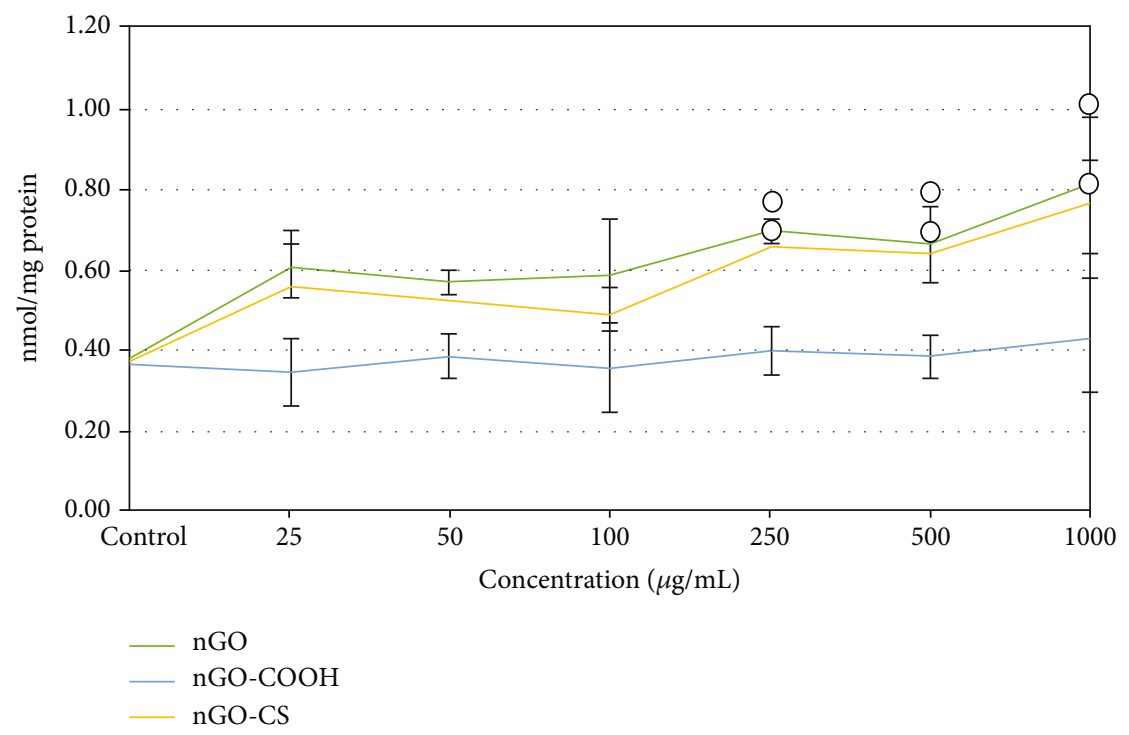

FIGURE 10: MDA concentration in the hDPSCs after $24 \mathrm{~h}$ incubation with different concentrations of graphene-based nanomaterials (25, 50, $100,250,500$, and $1000 \mu \mathrm{g} / \mathrm{mL}$ ). The MDA results are given in $\mathrm{nmol} / \mathrm{mg}$ protein. Results are the mean \pm SEM (bars) of three independent experiments each carried out in triplicate. $\mathrm{nGO}-\mathrm{COOH}$ did not significantly increase the intracellular MDA concentration at any concentration compared to the control group ( $p$ value $>0.05)$. ${ }^{*} p$ value $<0.05$.

robust than the hydroxyl bonds. Thus, carboxylation of nGO can create a more biocompatible nanosheet with excellent colloidal stability. Some studies on other carbon-based nanomaterials, such as carbon nanotubes, revealed that carboxylation could make them more cytocompatible $[43,44]$.

In this research, we investigated the effects of nGO, nGO$\mathrm{CS}$, and $\mathrm{nGO}-\mathrm{COOH}$ on $\mathrm{LDH}, \mathrm{GSH}$, SOD, and MDA that we found varying results.
The LDH activity assay was done to evaluate the effect of graphene nanosheets on the cell membrane integrity of hDPSCs. The results demonstrated that nGO exposure caused membrane damage, and LDH leakage was increased in a dose-dependent manner (Figure 7). Although exposure to nGO-CS leads to lower $\mathrm{LDH}$ leakage than nGO, nGO$\mathrm{COOH}$ had no significant effect on LDH leakage in hDPSCs ( $p$ value $>0.05$ ). These findings are in line with the results of 


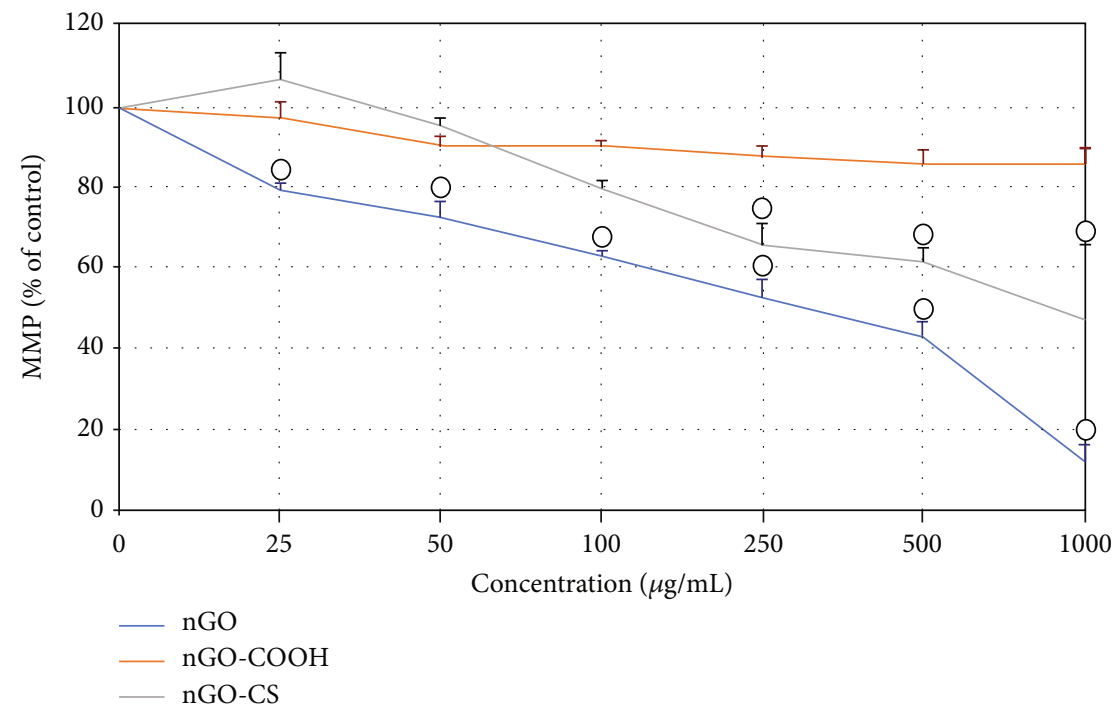

FIGURE 11: MMP in the hDPSCs after $24 \mathrm{~h}$ incubation with different concentrations of graphene-based nanomaterials (25, 50, 100, 250, 500, and $1000 \mu \mathrm{g} / \mathrm{mL}$ ). The results are given in percent compared to the untreated control group. Results are the mean \pm SEM (bars) of three independent experiments each carried out in triplicate. ${ }^{*} p$ value $<0.05$.

Sasidharan et al. [45] and Zhang et al. [46], which reported that high concentrations of graphene could damage the membrane. In a study, Chang et al. did not found any undesirable effect of GO nanoplatelets with a concentration of $\geq 50 \mu \mathrm{g} / \mathrm{mL}$ on the plasma membrane in the human lung cell line [40]. Contrary to these results, Lammel et al. have reported that the graphene family tends to bind to the cell and organelle membranes and, even at low concentrations, can disrupt their integrity [47]. Liao et al. also showed that both graphene and $\mathrm{nGO}$ sheets were capable of disorganizing in the plasma membrane [34]. The difference between our results and other results could be due to a discrepancy in the size of the test platelets, the assay protocol, and the sensitivity of the cells.

Furthermore, we investigated the effect of nGO and its functionalized derivatives on SOD activity in hDPSCs. The results showed that nGO significantly lowered the SOD activity compared to the control ( $p$ value $<0.05$ ) (Figure 9). The lowest decrease in SOD activity has been seen in the cell, which is exposed to nGO-COOH. Although nGO-CS displayed a lower reduction in superoxide dismutase activity compared to nGO, it still caused a significant decrease compared to the control group, especially at high concentrations. Besides, there was a significant decrease in the level of GSH in the nGO group compared to the control. In contrast, cell exposure to nGO-COOH did not lead to GSH depletion, and CS-nGO had an intermediate effect. These results revealed that the oxidative stress level was elevated in the nGO-treated cells. In agreement with our results, there are various studies on the toxicity of graphene in cells. For example, in a study, Gurunathan et al. indicated that nGO reduced the rate of GSH and SOD compared to the control group [48]. Also, Yuan and Gurunathan showed that nGO derivatives reduced GSH and SOD levels compared to the control group [49]. Also, in a study, Wang et al. displayed that GO reduced the rate of SOD [50]. In addition, nGO-COOH did not significantly increase the intracellular MDA concentration at any concentration compared to the control group ( $p$ value $>0.05$ ). The nGO-treated group at 500 and $1000 \mu \mathrm{g} / \mathrm{mL}$ resulted in the highest MDA concentration, approximately twice the nGO-COOH level group. These results are in agreement with those previous studies [51, 52], which revealed that some graphene-based nanomaterials, including $\mathrm{nGO}$, intensified the intracellular oxidative stress in a time and dose-dependent pattern. Also, in a study, Gurunathan et al. revealed that MDA levels increased after exposure to nGO derivatives [48]. In addition, Yuan and Gurunathan exhibited that nGO derivatives increased MDA levels compared to the control group [49]. In this study, it was found that at a high concentration $(1000 \mu \mathrm{g} / \mathrm{mL})$ of nGO, MMP was reduced to $12.3 \%$ of the control group in the hDPSCs. nGO disturbed the MMP of hDPSCs significantly more than the control group, and nGO-COOH did not impair it even at high concentrations $(85.59 \%$ of the control group). In comparison, nGO-CS disrupted the MMP only at high concentrations (47.27\% of the control group) (Figure 11). In previous studies, some researchers revealed that some graphene derivatives could reduce MMP of the macrophage cell line in a time- and concentrationdependent manner $[53,54]$. It seems that two important mechanisms are involved in causing damage to mitochondria: (a) direct and physical interactions between nGOs and mitochondrial and/or lysosomal membranes and consequently structural degradation [41] and (b) creating oxidative stress for mitochondrial infrastructures resulting in functional impairment $[55,56]$. Gurunathan et al. [48] and Yuan and Gurunathan [49] revealed that MMP levels reduced after exposure to nGO derivatives. Conclusively, although nGO has been extensively attracting in biotechnology and biomedicine, its cytotoxicity had remained a significant concern. Here, according to comprehensive cytotoxicity assays, $\mathrm{IC}_{50}$ of nGO, nGO-CS, and nGO-COOH were 232.01, 
467.61 , and $\geq 1000 \mu \mathrm{g} / \mathrm{mL}$, respectively. Our data confirm that carboxylation of $\mathrm{nGO}$ provides a lattice with better biocompatibility. In addition to reducing the cytotoxicity, carboxylation makes $\mathrm{nGO}$ a suitable carrier for loading many low-soluble drugs, especially anticancer agents [27, 57, 58]. As nGO-COOH is concentrated in intracellular vesicles, it can provide a promising platform for drug delivery.

\section{Conclusion}

Results illustrated nGO-CS more cytocompatible than nGO but not as safe as nGO-COOH. The most biocompatible derivative in this study was $\mathrm{nGO}-\mathrm{COOH}$, synthesized via a secure and cost-effective method that introduced it as a better candidate than $\mathrm{nGO}$ and $\mathrm{nGO}-\mathrm{CS}$ for bioapplications.

The results of this research demonstrated the lower toxicity and higher cytocompatibility of nGO-CS and nGO$\mathrm{COOH}$ compared to nGO. nGO-COOH not only has any adverse effect on the cell membrane and mitochondrial activity but also shows slight antioxidant activity at some concentrations. The findings help design safe and cytocompatible nGO derivatives for biomedical applications in dental fields.

Despite this study, further investigations are still needed to determine the long-term toxicity potential of these nanomaterials on dental cells and tissues along with their effects on different tissues and organs, especially cells in the oral cavity. Also, an in-depth evaluation of the mechanisms and processes involved in the osteogenic effect of the graphene family and the identification of intracellular signaling and metabolic pathways are needed in the future. Eventually, our study revealed that when deeply explored, the use of well-characterized, surface-modified graphene nanosheets leads to even more reliable dental treatments in the next years.

\section{Data Availability}

After publishing the article, any reader can access the data supporting the conclusions of the study by mailing us or the journal. The journal, reviewer, or whoever that is introduced by the journal to us can have data. Our data is fully available.

\section{Conflicts of Interest}

The authors declare that they have no conflict of interest.

\section{Authors' Contributions}

A.G. performed the experiments. A.G., F.E., A.A., M.S., M.C., and N.O. participated in the interpretation of results. All authors reviewed the paper.

\section{Acknowledgments}

The authors acknowledge the Biotechnology Research Center and Pharmaceutical Sciences Research Center, Shiraz University of Medical Sciences, for the financial support for this study.

\section{References}

[1] W. Zhang and P. C. Yelick, "Vital pulp therapy-current progress of dental pulp regeneration and revascularization," International Journal of Dentistry, vol. 2010, Article ID 856087, 9 pages, 2010.

[2] A. Ghanbarzadegan, M. Ajami, and M. Aminsobhani, "The effect of different combinations of calcium hydroxide as intra-canal medicament on endodontic pain: a randomized clinical trial study," Iranian Endodontic Journal, vol. 14, no. 1, pp. 1-6, 2018.

[3] A. Abbaszadegan, S. Dadolahi, A. Gholami et al., "Antimicrobial and cytotoxic activity of Cinnamomum zeylanicum, Calcium Hydroxide, and triple antibiotic paste as root canal dressing materials," The Journal of Contemporary Dental Practice, vol. 17, no. 2, pp. 105-113, 2018.

[4] K. P. Loh, Q. Bao, G. Eda, and M. Chhowalla, "Graphene oxide as a chemically tunable platform for optical applications," Nature Chemistry, vol. 2, no. 12, pp. 1015-1024, 2010.

[5] A. K. Geim, "Graphene: status and prospects," Science, vol. 324, no. 5934, pp. 1530-1534, 2009.

[6] M. H. Ghanbari, A. Khoshroo, H. Sobati, M. R. Ganjali, M. Rahimi-Nasrabadi, and F. Ahmadi, "An electrochemical sensor based on poly (L-cysteine)@AuNPs@ reduced graphene oxide nanocomposite for determination of levofloxacin," Microchemical Journal, vol. 147, pp. 198-206, 2019.

[7] M. H. Ghanbari, F. Shahdost-Fard, H. Salehzadeh et al., "A nanocomposite prepared from reduced graphene oxide, gold nanoparticles and poly (2-amino-5-mercapto-1, 3, 4-thiadiazole) for use in an electrochemical sensor for doxorubicin," Microchimica Acta, vol. 186, no. 9, 2019.

[8] R. Guazzo, C. Gardin, G. Bellin et al., "Graphene-based nanomaterials for tissue engineering in the dental field," Nanomaterials, vol. 8, no. 5, p. 349, 2018.

[9] R. Eivazzadeh-Keihan, A. Maleki, M. de la Guardia et al., "Carbon based nanomaterials for tissue engineering of bone: building new bone on small black scaffolds: a review," Journal of Advanced Research, vol. 18, pp. 185-201, 2019.

[10] Z. Ge, L. Yang, F. Xiao et al., "Graphene family nanomaterials: properties and potential applications in dentistry," International Journal of Biomaterials., vol. 2018, article 1539678, 12 pages, 2018.

[11] E. Nishida, H. Miyaji, A. Kato et al., "Graphene oxide scaffold accelerates cellular proliferative response and alveolar bone healing of tooth extraction socket," International Journal of Nanomedicine, vol. 11, pp. 2265-2277, 2016.

[12] J. H. Ahn, I.-R. Kim, Y. Kim et al., "The effect of mesoporous bioactive glass nanoparticles/graphene oxide composites on the differentiation and mineralization of human dental pulp stem cells," Nanomaterials, vol. 10, no. 4, p. 620, 2020.

[13] F. Gandomi, S. M. Peymani-Motlagh, M. Rostami et al., "Simple synthesis and characterization of $\mathrm{Li} 0.5 \mathrm{Fe} 2.5 \mathrm{O} 4$, $\mathrm{LiMg} 0.5 \mathrm{Fe} 2 \mathrm{O} 4$ and $\mathrm{LiNi} 0.5 \mathrm{Fe} 2 \mathrm{O} 4$, and investigation of their photocatalytic and anticancer properties on hela cells line," Journal of Materials Science: Materials in Electronics, vol. 30, no. 22, pp. 19691-19702, 2019.

[14] M. A. Marsooli, M. Fasihi-Ramandi, K. Adib et al., "Preparation and characterization of magnetic $\mathrm{Fe} 3 \mathrm{O} 4 / \mathrm{CdWO} 4$ and $\mathrm{Fe} 3 \mathrm{O} 4 / \mathrm{CdWO} 4 / \mathrm{PrVO} 4$ nanoparticles and investigation of their photocatalytic and anticancer properties on PANC1 cells," Materials, vol. 12, no. 19, p. 3274, 2019. 
[15] S. M. Peymani-Motlagh, A. Sobhani-Nasab, M. Rostami et al., "Assessing the magnetic, cytotoxic and photocatalytic influence of incorporating $\mathrm{Yb} 3+$ or Pr3+ ions in cobalt-nickel ferrite," Journal of Materials Science: Materials in Electronics, vol. 30, no. 7, pp. 6902-6909, 2019.

[16] F. Emadi, A. Amini, Y. Ghasemi, and A. Gholami, "Graphene: recent advances in engineering, medical and biological sciences, and future prospective," Trends in Pharmaceutical Sciences, vol. 4, no. 3, pp. 131-138, 2018.

[17] F. Emadi, A. Emadi, and A. Gholami, "A comprehensive insight towards pharmaceutical aspects of graphene nanosheets," Current Pharmaceutical Biotechnology, vol. 21, 2020.

[18] S. Bengtson, K. B. Knudsen, Z. O. Kyjovska et al., "Differences in inflammation and acute phase response but similar genotoxicity in mice following pulmonary exposure to graphene oxide and reduced graphene oxide," PLoS One, vol. 12, no. 6, article e0178355, 2017.

[19] N. A. El-Yamany, F. F. Mohamed, T. A. Salaheldin, A. A. Tohamy, W. N. Abd El-Mohsen, and A. S. Amin, "Graphene oxide nanosheets induced genotoxicity and pulmonary injury in mice," Experimental and Toxicologic Pathology, vol. 69, no. 6, pp. 383-392, 2017.

[20] S. Behvandi, A. Sobhani-Nasab, M. A. Karimi et al., "Synthesis and characterization of $\mathrm{Sm} 2(\mathrm{MoO} 4) 3, \mathrm{Sm} 2(\mathrm{MoO} 4) 3 / \mathrm{GO}$ and $\mathrm{Sm} 2(\mathrm{MoO} 4) 3 / \mathrm{C} 3 \mathrm{~N} 4$ nanostructures for improved photocatalytic performance and their anticancer the MCF-7 cells," Polyhedron, vol. 180, p. 114424, 2020.

[21] W. Asghar, H. Shafiee, V. Velasco et al., "Toxicology study of single-walled carbon nanotubes and reduced graphene oxide in human sperm," Scientific Reports, vol. 6, no. 1, article 30270, 2016.

[22] X. Guo and N. Mei, "Assessment of the toxic potential of graphene family nanomaterials," Journal of Food and Drug Analysis, vol. 22, no. 1, pp. 105-115, 2014.

[23] Z. Liu, J. T. Robinson, X. Sun, and H. Dai, "PEGylated nanographene oxide for delivery of water-insoluble cancer drugs," Journal of the American Chemical Society, vol. 130, no. 33, pp. 10876-10877, 2008.

[24] L. Zhang, J. Xia, Q. Zhao, L. Liu, and Z. Zhang, "Functional graphene oxide as a nanocarrier for controlled loading and targeted delivery of mixed anticancer drugs," Small, vol. 6, no. 4, pp. 537-544, 2010.

[25] A. Sahu, W. I. Choi, and G. Tae, "A stimuli-sensitive injectable graphene oxide composite hydrogel," Chemical Communications, vol. 48, no. 47, pp. 5820-5822, 2012.

[26] E. Bidram, A. Sulistio, A. Amini et al., "Fractionation of graphene oxide single nanosheets in water-glycerol solutions using gradient centrifugation," Carbon, vol. 103, pp. 363-371, 2016.

[27] A. Gholami, F. Emadi, M. Nazem et al., "Expression of key apoptotic genes in hepatocellular carcinoma cell line treated with etoposide-loaded graphene oxide," Journal of Drug Delivery Science and Technology, vol. 57, p. 101725, 2020.

[28] F. Emadi, A. Amini, A. Gholami, and Y. Ghasemi, "Functionalized graphene oxide with chitosan for protein nanocarriers to protect against enzymatic cleavage and retain collagenase activity," Scientific Reports, vol. 7, no. 1, article 42258, 2017.

[29] S. Yu, J. Liu, W. Zhu, Z. T. Hu, T. T. Lim, and X. Yan, "Facile room-temperature synthesis of carboxylated graphene oxidecopper sulfide nanocomposite with high photodegradation and disinfection activities under solar light irradiation," Scientific Reports, vol. 5, no. 1, article 16369, 2015.
[30] A. Abbaszadegan, A. Gholami, S. Abbaszadegan et al., "The effects of different ionic liquid coatings and the length of alkyl chain on antimicrobial and cytotoxic properties of silver nanoparticles," Iranian Endodontic Journal, vol. 12, no. 4, pp. 481487, 2017.

[31] N. Ebrahimi, S. Rasoul-Amini, A. Ebrahiminezhad, Y. Ghasemi, A. Gholami, and H. Seradj, "Comparative study on characteristics and cytotoxicity of bifunctional magneticsilver nanostructures: synthesized using three different reducing agents," Acta Metallurgica Sinica (English Letters), vol. 29, no. 4, pp. 326-334, 2016.

[32] A. Abbaszadegan, S. Sahebi, A. Gholami et al., "Timedependent antibacterial effects of Aloe vera and Zataria multiflora plant essential oils compared to calcium hydroxide in teeth infected with Enterococcus faecalis," Journal of Investigative and Clinical Dentistry, vol. 7, no. 1, pp. 93103, 2019.

[33] A. M. Dimiev and J. M. Tour, "Mechanism of graphene oxide formation," ACS Nano, vol. 8, no. 3, pp. 3060-3068, 2014.

[34] K.-H. Liao, Y.-S. Lin, C. W. Macosko, and C. L. Haynes, "Cytotoxicity of graphene oxide and graphene in human erythrocytes and skin fibroblasts," ACS Applied Materials \& Interfaces, vol. 3, no. 7, pp. 2607-2615, 2011.

[35] W. Hu, C. Peng, M. Lv et al., "Protein corona-mediated mitigation of cytotoxicity of graphene oxide," ACS Nano, vol. 5, no. 5, pp. 3693-3700, 2011.

[36] M. Wojtoniszak, X. Chen, R. J. Kalenczuk et al., "Synthesis, dispersion, and cytocompatibility of graphene oxide and reduced graphene oxide," Colloids and Surfaces B: Biointerfaces, vol. 89, pp. 79-85, 2012.

[37] L. Feng, S. Zhang, and Z. Liu, "Graphene based gene transfection," Nanoscale, vol. 3, no. 3, pp. 1252-1257, 2011.

[38] H. Pieper, S. Chercheja, S. Eigler, C. E. Halbig, M. R. Filipovic, and A. Mokhir, "Endoperoxides revealed as origin of the toxicity of graphene oxide," Angewandte Chemie International Edition, vol. 55, no. 1, pp. 405-407, 2016.

[39] Z. S. Singh, "Applications and toxicity of graphene family nanomaterials and their composites," Nanotechnology, Science and Applications, vol. 9, pp. 15-28, 2016.

[40] Y. Chang, S.-T. Yang, J.-H. Liu et al., "In vitro toxicity evaluation of graphene oxide on A549 cells," Toxicology Letters, vol. 200, no. 3, pp. 201-210, 2011.

[41] A. B. Seabra, A. J. Paula, R. de Lima, O. L. Alves, and N. Durán, "Nanotoxicity of graphene and graphene oxide," Chemical Research in Toxicology, vol. 27, no. 2, pp. 159-168, 2014.

[42] C. Karuppiah, S. Cheemalapati, S.-M. Chen, and S. Palanisamy, "Carboxyl-functionalized graphene oxidemodified electrode for the electrochemical determination of nonsteroidal anti-inflammatory drug diclofenac," Ionics, vol. 21, no. 1, pp. 231-238, 2015.

[43] S. Sweeney, S. Hu, P. Ruenraroengsak et al., "Carboxylation of multiwalled carbon nanotubes reduces their toxicity in primary human alveolar macrophages," Environmental Science: Nano, vol. 3, no. 6, pp. 1340-1350, 2016.

[44] Z. Liu, X. Dong, L. Song et al., "Carboxylation of multiwalled carbon nanotube enhanced its biocompatibility with L02 cells through decreased activation of mitochondrial apoptotic pathway," Journal of Biomedical Materials Research Part A, vol. 102, no. 3, pp. 665-673, 2014.

[45] A. Sasidharan, L. S. Panchakarla, P. Chandran et al., "Differential nano-bio interactions and toxicity effects of pristine versus 
functionalized graphene," Nanoscale, vol. 3, no. 6, pp. 24612464, 2011.

[46] Y. Zhang, S. F. Ali, E. Dervishi et al., "Cytotoxicity effects of graphene and single-wall carbon nanotubes in neural phaeochromocytoma-derived PC12 cells," ACS Nano, vol. 4, no. 6 , pp. 3181-3186, 2010.

[47] T. Lammel, P. Boisseaux, M.-L. Fernández-Cruz, and J. M. Navas, "Internalization and cytotoxicity of graphene oxide and carboxyl graphene nanoplatelets in the human hepatocellular carcinoma cell line Hep G2," Particle and Fibre Toxicology, vol. 10, no. 1, p. 27, 2013.

[48] S. Gurunathan, M.-H. Kang, M. Jeyaraj, and J.-H. Kim, "Differential immunomodulatory effect of graphene oxide and vanillin-functionalized graphene oxide nanoparticles in human acute monocytic leukemia cell line (THP-1)," International Journal of Molecular Sciences, vol. 20, no. 2, p. 247, 2019.

[49] Y.-G. Yuan and S. Gurunathan, "Combination of graphene oxide-silver nanoparticle nanocomposites and cisplatin enhances apoptosis and autophagy in human cervical cancer cells," International Journal of Nanomedicine, vol. 12, pp. 6537-6558, 2017.

[50] A. Wang, K. Pu, B. Dong et al., "Role of surface charge and oxidative stress in cytotoxicity and genotoxicity of graphene oxide towards human lung fibroblast cells," Journal of Applied Toxicology, vol. 33, no. 10, pp. 1156-1164, 2013.

[51] X. Zhang, W. Hu, J. Li, L. Tao, and Y. Wei, “A comparative study of cellular uptake and cytotoxicity of multi-walled carbon nanotubes, graphene oxide, and nanodiamond," Toxicology Research, vol. 1, no. 1, pp. 62-68, 2012.

[52] J. Wu, R. Yang, L. Zhang, Z. Fan, and S. Liu, "Cytotoxicity effect of graphene oxide on human MDA-MB-231 cells," Toxicology Mechanisms and Methods, vol. 25, no. 4, pp. 312-319, 2015.

[53] J. Szczepaniak, B. Strojny, E. S. Chwalibog et al., "Effects of reduced graphene oxides on apoptosis and cell cycle of glioblastoma multiforme," International Journal of Molecular Sciences, vol. 19, no. 12, p. 3939, 2018.

[54] S. X.-F. Adamson, R. Wang, W. Wu, B. Cooper, and J. Shannahan, "Metabolomic insights of macrophage responses to graphene nanoplatelets: Role of scavenger receptor CD36," PLoS One, vol. 13, no. 11, article e0207042, 2018.

[55] A. Piperno, A. Scala, A. Mazzaglia et al., "Cellular signaling pathways activated by functional graphene nanomaterials," International Journal of Molecular Sciences, vol. 19, no. 11, article 3365, 2018.

[56] S. Jaworski, B. Strojny, E. Sawosz et al., "Degradation of mitochondria and oxidative stress as the main mechanism of toxicity of pristine graphene on U87 glioblastoma cells and tumors and HS-5 cells," International Journal of Molecular Sciences, vol. 20, no. 3, p. 650, 2019.

[57] C. McCallion, J. Burthem, K. Rees-Unwin, A. Golovanov, and A. Pluen, "Graphene in therapeutics delivery: problems, solutions and future opportunities," European Journal of Pharmaceutics and Biopharmaceutics, vol. 104, pp. 235-250, 2016.

[58] S. Shi, F. Chen, E. B. Ehlerding, and W. Cai, "Surface engineering of graphene-based nanomaterials for biomedical applications," Bioconjugate Chemistry, vol. 25, no. 9, pp. 1609-1619, 2014. 